\title{
IFITMs Inhibit Cell Fusion Mediated by Trophoblast Syncytins
}

Ashley Zani ${ }^{1,4}$, Lizhi Zhang ${ }^{1,4}$, Adam Kenney ${ }^{1,4}$, Temet M. McMichael ${ }^{1,4}$, Jesse J. Kwiek ${ }^{2,4}$, ShanLu Liu ${ }^{3,4}$, and Jacob S. Yount ${ }^{1,4, *}$

${ }^{1}$ Department of Microbial Infection and Immunity, The Ohio State University, Columbus OH

${ }^{2}$ Department of Microbiology, The Ohio State University, Columbus, $\mathrm{OH}$

${ }^{3}$ Department of Veterinary Biosciences, The Ohio State University, Columbus, $\mathrm{OH}$

${ }^{4}$ Infectious Diseases Institute, The Ohio State University, Columbus $\mathrm{OH}$

*Correspondence: jacob.yount@osumc.edu

\section{Abstract}

Type I interferon (IFN) induced by virus infections during pregnancy causes placental damage, though the mechanisms and identities of IFN-stimulated genes that are involved remain under investigation. The IFN-induced transmembrane proteins (IFITMs) inhibit virus infections by preventing virus membrane fusion with cells and by inhibiting fusion of infected cells (syncytialization). Fusion of placental trophoblasts via expression of endogenous retroviral fusogens known as Syncytins forms the syncytiotrophoblast, a multinucleated cell structure essential for fetal development. We found that IFN blocks fusion of BeWo human placental trophoblasts. Stably-expressed IFITMs 1, 2, and 3 also blocked fusion of these trophoblasts, while making them more resistant to virus infections. Conversely, stable knockdown of IFITMs in BeWo trophoblasts increased their spontaneous fusion and allowed fusion in the presence of IFN, while also making the cells more susceptible to virus infection. Overall, our data demonstrate that IFITMs are anti-viral and anti-fusogenic in trophoblasts. 


\section{Main Text}

Infections during pregnancy are associated with low birth weights, congenital birth defects, pregnancy complications, and miscarriages (1). Placental damage has been observed during Zika virus infections in non-human primates (2), and in mice during Zika virus and other

flavivirus infections (3). Further, a recent landmark study demonstrated that fetal mortality during Zika virus infection is mediated by type I interferon (IFN) receptor signaling(4). This paradoxical effect of what are considered to be beneficial antiviral cytokines may be an evolutionary adaptation in which IFNs signal for termination of pregnancies that are unlikely to be viable due to severe or prolonged infection $(4,5)$. IFN signaling was shown to disrupt placental architecture in mice, leading to fetal hypoxia and demise. Placental defects caused by IFNs included disruption of the syncytiotrophoblast, a multinucleated cell structure that is critical for nutrient and gas exchange between maternal and fetal blood and that is formed by cell-to-cell fusion of trophoblasts. Though IFNs have been known for decades to be embryotoxic molecules (6-9), the mechanism by which they induce placental damage is not fully understood.

Our laboratory has long studied the IFN-induced transmembrane proteins (IFITMs) 1, 2, and 3, which utilize a palmitoylated amphipathic helical domain to block membrane fusion between viruses and host cells through alteration of lipid bilayers (10-16). These proteins are also capable of blocking cell-to-cell fusion, i.e., syncytia formation, of infected cells $(11,12,17)$. Given that placental trophoblast fusion is mediated by unique expression of endogenous retroviral fusion proteins known as Syncytin-1 and -2 (18-23), we hypothesized that IFN and specifically the IFITMs, could inhibit trophoblast fusion and may thus underlie the embryotoxicity of IFN. We sought to test this hypothesis using the human BeWo trophoblast cell line, one of the most commonly used model systems for studying Syncytin-mediated trophoblast fusion (21-27). These cells have a low baseline level of spontaneous fusion, but increase production of Syncytins upon stimulation with forskolin, resulting in robust cell-to-cell fusion (21-26). 
Similar to most other cell types, BeWo cells and primary trophoblasts have been reported to produce low steady state levels of IFITM mRNAs even in the absence of IFN (2830). However, IFITM 1, 2, or 3 were not detected by Western blotting in BeWo cells, indicating that baseline protein levels are low (Fig 1A). Upon IFN treatment, IFITMs 1-3, ISG15, and RIG-I were upregulated, demonstrating functional IFN signaling in these cells (Fig 1A). Co-treatment with fusion-inducing forskolin had no effect on levels of the IFN-induced proteins (Fig $1 \mathrm{~A})$. In these treated cells, we quantified cell-to-cell fusion and observed that forskolin treatment significantly increased the fusion index of the cells as expected (Fig 1B,C). This fusion was significantly decreased by IFN co-treatment, demonstrating that type I IFN inhibits fusion of BeWo placental trophoblasts concomitant with induction of IFN-induced proteins, such as IFITMs (Fig 1A-C). The overall level of E-cadherin generally decreases in fused BeWo cells, and was thus moderately decreased in forskolin-treated cells as measured by Western blotting, but was not decreased in forskolin/IFN co-treated cells, providing a secondary indicator of fusion inhibition by IFN (Fig 1B).

To more specifically examine the effect of the individual IFITMs on trophoblast fusion, we generated stable BeWo lines expressing IFITMs 1, 2, or 3, or an IFITM3 variant (Y20A) that is reported to have decreased endocytic capacity and thus accumulates at the plasma membrane $(31,32)$ (Fig 2A). To test whether IFITMs are antivirally functional in BeWo cells, we examined infections of the cell lines with influenza A virus and Zika virus. We found that each of the IFITMs decreased infection with influenza virus as compared to vector control cells, and that IFITMs 1 and 3 provided protection against Zika virus infection (Fig 2B). We further observed that forskolin was unable to induce fusion of the IFITM-expressing BeWo lines (Fig 2C,D), indicating that IFITMs 1, 2, and 3 are each capable of inhibiting trophoblast fusion. Thus, IFITM expression in trophoblasts decreases both virus infection and cell-to-cell fusion. 
In order to determine whether IFITMs are specifically capable of inhibiting fusion mediated individually by Syncytin-1 or -2, we employed a cell-to-cell fusion assay utilizing HEK293T cells, which naturally lack expression of endogenous fusion proteins and endogenous IFITMs, and that we previously validated for studying effects of IFITMs on cell-to-cell fusion mediated by viral fusion proteins $(12,17,33)$. In short, one population of cells was transfected with vector control or Syncytin-1 or -2 , while target cells were transfected with individual IFITM expression plasmids or vector control. Additionally, the two groups of cells were co-transfected with distinct plasmids that produce luciferase only when the plasmids are together in the same cell (34) (Fig 3A). Thus, after mixing the two cell populations, luciferase activity serves as a quantitative readout of cell-to-cell fusion between the two populations $(12,17,34)$. We first established that both Syncytin-1 and -2 were able to induce fusion of HEK293T cells (Fig 3B). Fusion mediated by either Syncytin was significantly inhibited by expression of IFITMs in target cells, further establishing that IFITMs are capable of inhibiting cell-to-cell fusion mediated by these essential trophoblast fusogens (Fig 3B). Further, these data demonstrate that inhibition of Syncytin-mediated cell fusion by IFITMs does not require intracellular interaction between Syncytins and IFITMs as these proteins were expressed in distinct cell populations in these experiments.

To determine whether endogenous levels of IFITMs are capable of inhibiting trophoblast fusion, we generated stable BeWo cell lines expressing shRNAs targeting the IFITMs. We generated two cell lines (labeled shIFITM \#1 and \#3) with significant knockdowns of IFITMs 1-3, resulting in low IFITM levels as compared to control cells even with IFN treatment (Fig 4A). Surprisingly, we found that these cells were difficult to maintain when using standard BeWo passaging methods, but that the cells could be readily expanded when passaged frequently at high dilution. We determined that this was likely due to increased spontaneous fusion of these cells and thus their terminal differentiation when grown at high density. We thus quantified fusion of these cells when plated at high density, and found that increased spontaneous fusion 
was indeed occurring for both of the IFITM knockdown cell lines as compared to control cells, and this fusion was not increased further by forskolin treatment (Fig 4B,C). Strikingly, ECadherin was downregulated under these conditions more than we have observed with forskolin treatments, providing a secondary confirmation of the robust spontaneous fusion of these IFITM knockdown BeWo cells (Fig 4D). These results suggest that BeWo cells are capable of fusing without forskolin treatment, but that this is prevented by a low baseline level of IFITMs. Indeed, we were able to detect basal IFITM3 in WT BeWo cells when we prevented IFITM3 turnover in lysosomes by treatment of cells with chloroquine (35) (Fig 4E). Additionally, we observed that IFN was unable to inhibit the spontaneous fusion of these cells, identifying that inhibition of trophoblast fusion by IFN requires the IFITMs (Fig 4F,G). Finally, we measured the Zika virus infection rates of control and IFITM knockdown cells and saw increased infection in the absence of IFITMs (Fig 4H). Overall, we have employed gain- and loss-of-function experiments to identify that IFITMs inhibit virus infection of trophoblasts while also inhibiting critical cell-to-cell fusion required for syncytiotophoblast formation.

Together, the results presented here have significant implications for our understanding of the toxic effects of IFNs in infections during pregnancy since they suggest that IFITMs are a critical contributor to this toxicity through effects on placenta syncytiotrophoblast development. The IFITMs may provide antiviral protection during moderate pregnancy-associated infections, including protection against direct viral infections of trophoblasts or the syncytiotrophoblast. However, we posit that IFITMs may also provide a sustained disruption of syncytiotrophoblast formation/maintenance during severe or lengthy infections, resulting in fetal demise. Our data corroborate a groundbreaking recent study showing that IFITMs are required for placental malformations and fetal resorption induced by the viral mimic Polyl:C in mice (27). These observations may also explain the need for tight regulation of baseline cellular levels of IFITM3 by posttranslational modifications, including phosphorylation and ubiquitination, in the absence of infection $(14,31,35)$. Interestingly, the antiviral activity of IFITMs is counteracted by the 
clinically approved drug amphotericin B $(17,36)$, suggesting that this drug may also hold promise for countering the negative effects of IFITMs on the placenta. Thus, the identification of the IFITMs as a missing link between IFN and placental disruption may provide specific molecular targets for mitigating negative effects of IFNs during pregnancy-associated infections.

\section{Materials and Methods}

\section{Cell culture and virus infections}

An initial stock of Bewo cells was kindly provided by Dr. Stephanie Seveau (The Ohio State University). We confirmed the identity of these cells by confirming their expression of Syncytins and by confirming their fusion in the presence of forskolin. BeWo cells were cultured in Ham's F12K media supplemented with 10\% Equafetal Bovine Serum (Atlas Biologicals). HEK293T and Vero cells were purchased from the ATCC and were grown in DMEM supplemented with $10 \%$ Equafetal Bovine Serum. All cells were cultured in a humidified incubator at $37^{\circ} \mathrm{C}$ with $5 \% \mathrm{CO}_{2}$ and were confirmed to be free of mycoplasma contamination by spot checking with the Lonza MycoAlert Mycoplasma Detection Kit. In some experiments, BeWo cells were treated with IFN $\beta$ (EMD Millipore) at a concentration of 40 units $/ \mathrm{mL}$. For infections, influenza virus A/PR/8/34 (H1N1) (provided by Dr. Thomas Moran of the Icahn School of Medicine at Mt. Sinai) was propagated in embryonated chicken eggs and titered as previously described (37), and Zika virus strain PRVABC59 (NIH BEI Resources) was propagated and titered in Vero cells. For detection of influenza virus infected cells, anti-nucleoprotein antibody (NIH BEI Resources, NR19868) staining was used. For detection of Zika virus infection, anti-Zika E antibody (Kerafast) was used. Percent infection was measured by flow cytometry with mock-infected cells serving as controls for gating. Samples were analyzed using FlowJo software.

\section{Generation of stable BeWo cell lines}


For stable expression of IFITM proteins, IFITM coding sequences were cloned into the pLentipuro vector as described previously (17). Lentiviruses were generated in HEK293T cells and were concentrated using Lenti-X Concentrator reagent (Takara Bio) before transduction of BeWo cells. Stable IFITM-expressing BeWo lines were selected and subsequently maintained with $1 \mathrm{ug} / \mathrm{mL}$ puromycin in the media. IFITM knockdown lines were generated and maintained similarly using lentiviral shRNA constructs purchased from Sigma as described previously (38).

\section{BeWo cell fusion assays}

For analysis of cell fusion, BeWo cells were plated to achieve approximately $75 \%$ confluence on glass slides. To induce fusion, 50 uM forskolin (Sigma) was added to culture media for 48-72 h. For imaging, cells were fixed with $4 \%$ paraformaldehyde, permeabilized with PBS $/ 0.1 \%$ TritonX100, and blocked with PBS/2\% Equafetal Bovine Serum. Cells were then stained with anti-ECadherin antibody (Abcam, ab1416) at 1:100 in PBS/0.1\% Triton-X100 followed by staining with Alexa Fluor 488-labeled goat anti-mouse secondary antibody (Life Technologies) at 1:1000 in PBS/0.1\% Triton-X100. Slides were mounted using Prolong Gold Antifade Mountant with DAPI. Cells were imaged on an Olympus FluoView Confocal Microscope. For each sample, 10 randomly chosen images were collected. In each field, nuclei in mono- versus multi-nucleated cells (two or more nuclei within a continuous E-Cadherin stained cell border) were counted. ECadherin staining was digitally enhanced using ImageJ software (NIH) to assist with identification of fused and unfused cells. The percentage of fused cells in each field was calculated as nuclei in multinucleated cells divided by the total number of nuclei in the field. Values for each of the ten fields were averaged to determine the percent fusion for each sample. Generally, more than 400 nuclei were counted for each sample. The percent fusion value for the negative control in each experiment (WT BeWo or vector transduced BeWo without forskolin) was set to 1 and all other samples were normalized to this value. The percent spontaneous fusion for the negative controls varied from roughly $10-20 \%$ in different 
experiments and likely represented cells that were fused prior to plating and cells that spontaneously fused during the experiment, and may have potentially also included a small number of false positive multinucleated cells. Fusion induced by forskolin or knockdown of the IFITMs was generally 2-3 fold above the negative control and represented values of roughly 30 $80 \%$ fusion in different experiments.

\section{HEK293T cell fusion assays}

IFITM constructs cloned into the pCMV-HA vector (Clontech) were described previously $(13,31)$ and myc-FLAG-tagged Syncytin-1 and 2 expression constructs in the pCMV6 vector were purchased from Origene. pFR-Luc and pBD-NFkB (Agilent) were co-transfected with plasmids as indicated in Fig 3A. Cell-to-cell fusion assays were performed as outlined and validated previously $(12,17,34)$. In short, transfections were performed overnight using LipoJet transfection reagent (Signagen). Cell populations were then mixed, replated, and incubated at $37^{\circ} \mathrm{C}$ in standard culture media for $24 \mathrm{~h}$. Luciferase activity was measured using the Promega Dual-Luciferase Reporter Assay System.

\section{Western blotting}

For Western blotting, cells were lysed in 1\% SDS buffer (1\% SDS, $150 \mathrm{mM} \mathrm{NaCl}, 50 \mathrm{mM}$ triethanolamine, $\mathrm{pH}$ 7.4), supplemented with cOmplete EDTA-Free Protease Inhibitor Cocktail (Sigma) at $5 x$ the recommended concentration. Western antibodies used in this study include those specific for the following proteins: E-Cadherin (Abcam), ISG15 (Cell Signaling), RIG-I (Cell Signaling), IFITM1 (Cell Signaling), IFITM3 (ProteinTech, detects both IFITM2 and IFITM3), and GAPDH (Life Technologies).

\section{Acknowledgments}


This work was supported by NIH grants Al130110 and Al142256 to JSY. Ashley Zani was supported by NIH training grant Al112542 administered by the OSU Infectious Diseases Institute. The authors thank Dr. Stephanie Seveau for supplying an initial stock of BeWo cells and providing technical advice for fusion assays. 
A

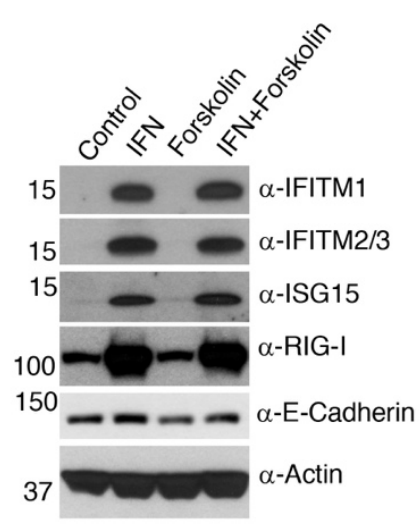

B
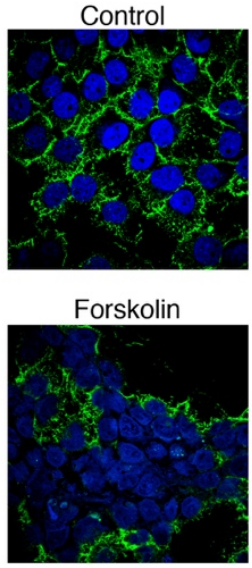

IFN

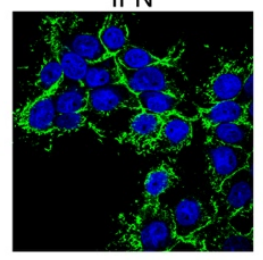

Forskolin + IFN

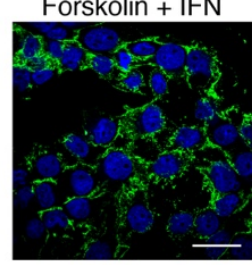

C

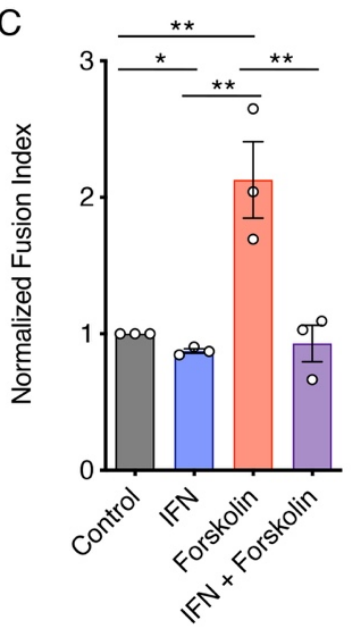

Figure 1: IFN inhibits fusion of BeWo trophoblasts. BeWo cells were treated for $24 \mathrm{~h}$ with 40 units/mL IFN $\beta$ (IFN), 50 uM forskolin, a combination of IFN and forskolin, or vehicle control. Treated cells were analyzed by A) Western blotting of cell lysates or B) Confocal microscopy imaging after staining with anti-E-Cadherin (green) or DAPI (blue). White scale bar, 10 um. C) Fusion indices were calculated and normalized to the background spontaneous fusion level observed in each experiment in vehicle control cells. Bars represent averages from three identical independent experiments with individual data points shown as circles, and data are representative of two additional similar experiments. Error bars represent standard deviation of the mean. ${ }^{*} p<0.05,{ }^{* *} p<0.01$ by paired t-test. 
A

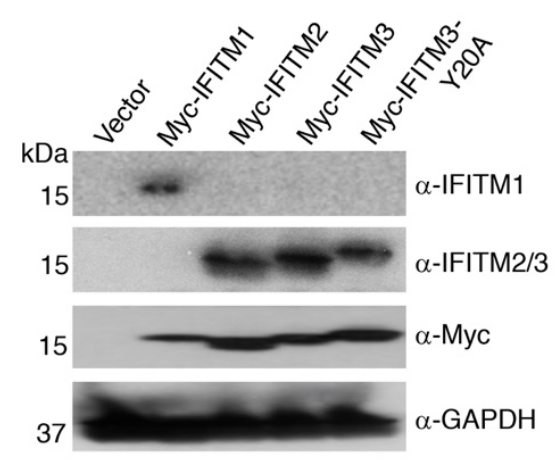

C
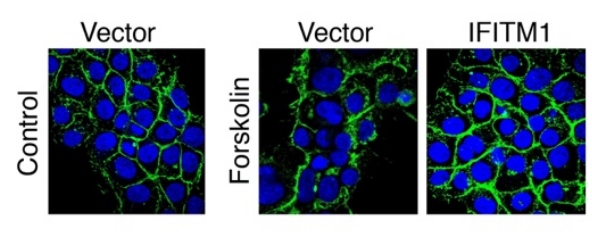

B
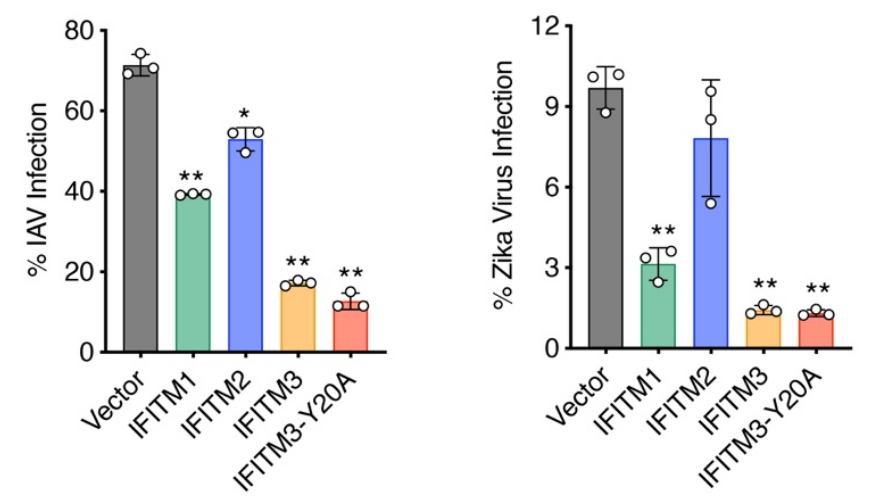

D

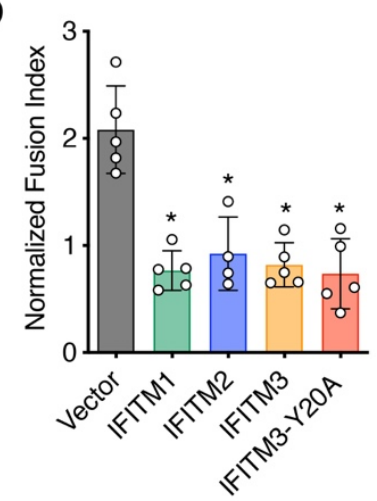

Figure 2: IFITM overexpression inhibits BeWo trophoblast fusion. BeWo cells were stably transduced with lentiviruses expressing myc-tagged IFITM constructs. A) Western blotting of cell lysates. B) Percent infection $24 \mathrm{~h}$ post infection with influenza A virus (IAV) (MOI 2.5) or Zika virus (MOI 5) as measured by flow cytometry staining for viral antigens. Flow cytometry gates were set based on mock infected controls. Bars represent averages of three independent experiments with individual data points shown as circles. Error bars represent standard deviation of the mean. ${ }^{*} p<0.05$, ${ }^{* *} p<0.01$ as compared to vector control cells by paired t-test. C) Cell lines were treated for $48 \mathrm{~h}$ with $50 \mathrm{uM}$ forskolin or vehicle control. Cells were then imaged by confocal microscopy after staining with anti-E-Cadherin (green) or DAPI (blue). White scale bar, 10 um. D) Fusion indices from forskolin-treated cells as in $\mathbf{C}$ were calculated and normalized to the background spontaneous fusion levels observed in each experiment in vector control cells treated with vehicle control. Bars represent averages from five independent experiments with individual data points shown as circles. Error bars represent standard deviation of the mean. ${ }^{*} p<0.05$ as compared to forskolin-treated vector control cells by paired $t$ test. 

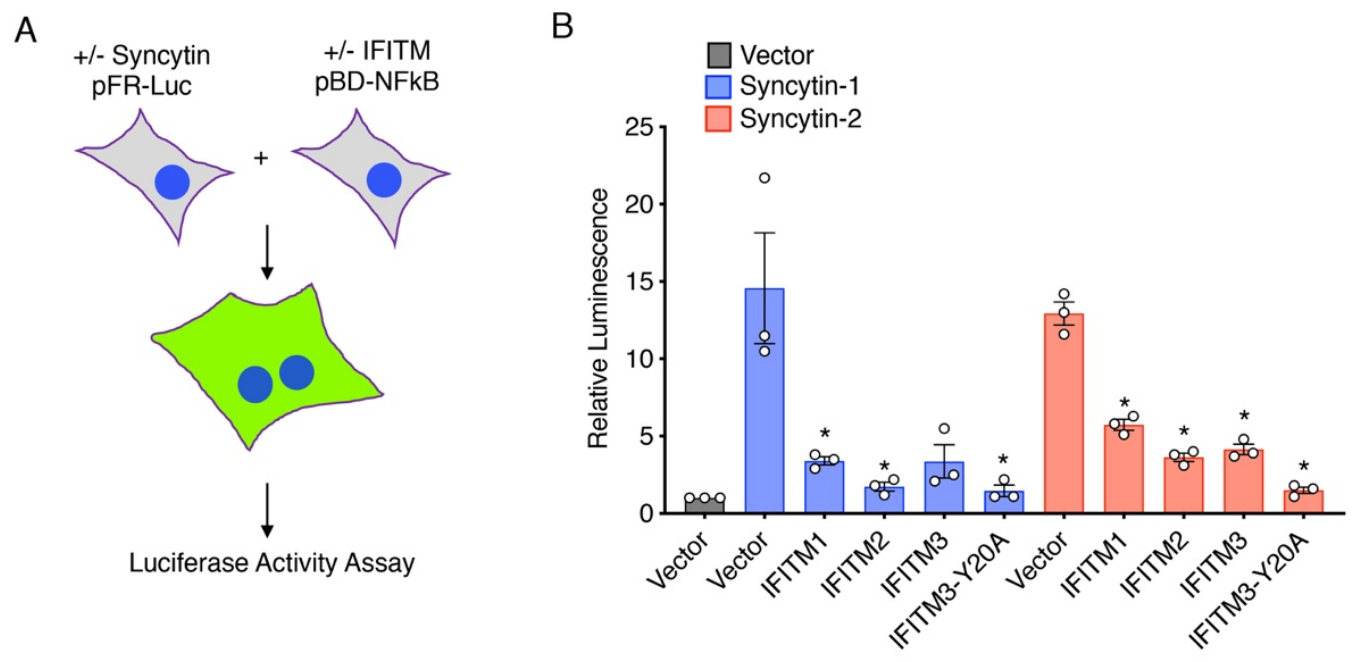

Figure 3: IFITMs inhibition Syncytin-mediated fusion of HEK293T cells. A) Schematic representation of the HEK293T cell fusion assay. One population of cells was transfected with Syncytin-1 or -2 constructs or vector control plus pFR-Luc plasmid. A second population of cells was transfected with IFITM constructs or vector control plus pBD-NFkB. Cells were then mixed and allowed to fuse for $24 \mathrm{~h}$. Luciferase activity in cell lysates was then measured as a quantitative readout of cell fusion. B) Luciferase activity was measured from cells prepared as described in A. Bars represent average results from three independent experiments with individual data points shown as circles. Error bars represent standard deviation of the mean. ${ }^{*} \mathrm{p}<0.05$ as compared to the respective vector controls by paired t-test. 

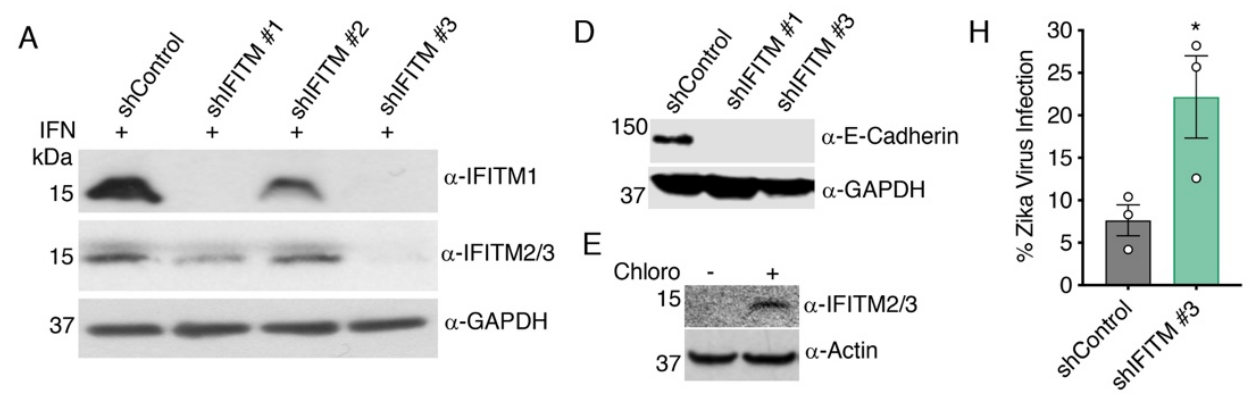

B

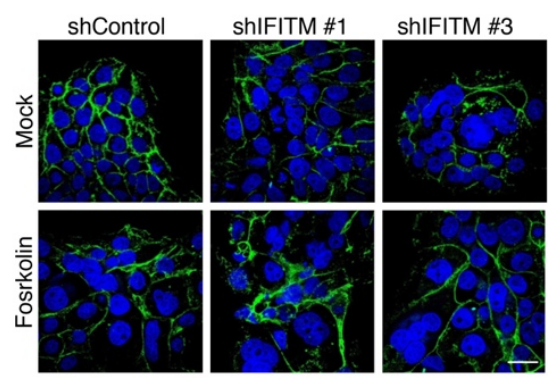

$\mathrm{F}$

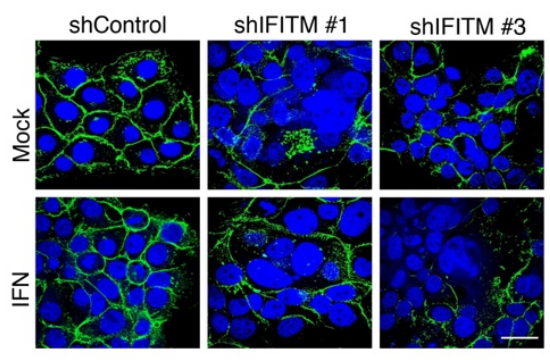

C

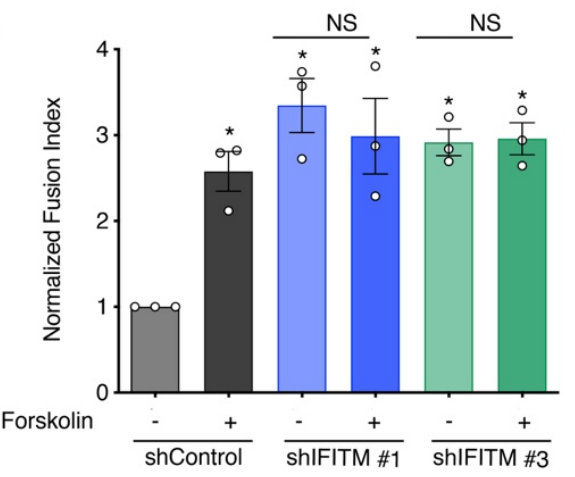

G

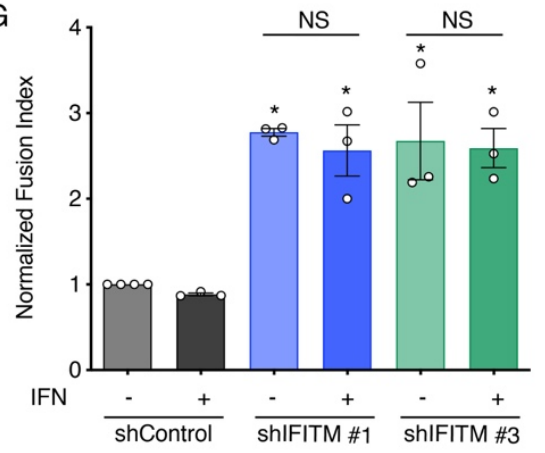

Figure 4: Knockdown of IFITMs promotes fusion of BeWo trophoblasts. BeWo cells were stably transduced with lentiviruses expressing control shRNA (shControl) or different shRNAs targeting IFITMs (shIFITM \#1-3). A) Western blotting of cell lysates after $18 \mathrm{~h}$ treatment with 40 units/mL IFN $\beta$ demonstrates IFITM knockdown in specific cell lines. B) The indicated cell lines were treated for $48 \mathrm{~h}$ with $50 \mathrm{uM}$ forskolin or vehicle control. Cells were then imaged by confocal microscopy after staining with anti-E-Cadherin (green) or DAPI (blue). White scale bar, $10 \mathrm{um}$. C) Fusion indices from cells as in B were calculated and normalized to the background spontaneous fusion levels observed in each experiment in shControl cells treated with vehicle control. Bars represent averages from three independent experiments with individual data points shown as circles. Error bars represent standard deviation of the mean. ${ }^{*} p<0.01$ as compared to shControl with vehicle control by paired t-test. NS, not significantly different. D) Western blotting of cell lysates demonstrates downregulation of E-Cadherin in IFITM knockdown lines. E) Western blotting of WT Bewo cells after $24 \mathrm{~h}$ treatment with $40 \mathrm{uM}$ chloroquine (chloro) or vehicle control (mock) demonstrates accumulation of IFITM2/3 when lysosomal degradation is inhibited. F) The indicated cell lines were treated for $48 \mathrm{~h}$ with 40 units $/ \mathrm{mL}$ IFN $\beta$ or vehicle control. Cells were then imaged by confocal microscopy after staining with anti-E-Cadherin (green) or DAPI (blue). White scale bar, $10 \mathrm{um}$. G) Fusion indices from cells as in $\mathbf{F}$ were calculated and normalized to the background spontaneous fusion levels observed in each 
experiment in shControl cells treated with vehicle control. Bars represent averages from three independent experiments with individual data points shown as circles. Error bars represent standard deviation of the mean. ${ }^{*} p<0.05$ as compared to shControl with vehicle control by paired t-test. NS, not significantly different. H) Percent infection of shControl cells and shIFITM \#3 cells $24 \mathrm{~h}$ post infection with Zika virus (MOI 5) as measured by flow cytometry staining for viral antigen. Flow cytometry gates were set based on mock infected controls. Bars represent averages of three independent experiments with individual data points shown as circles. Error bars represent standard deviation of the mean. ${ }^{*} p<0.05$ as compared to shControl cells by paired t-test.

\section{References}

1. Coyne CB, Lazear HM. 2016. Zika virus - reigniting the TORCH. Nat Rev Microbiol 14:707-715.

2. Hirsch AJ, Roberts VHJ, Grigsby PL, Haese N, Schabel MC, Wang X, Lo JO, Liu Z, Kroenke CD, Smith JL, Kelleher M, Broeckel R, Kreklywich CN, Parkins CJ, Denton M, Smith P, DeFilippis V, Messer W, Nelson JA, Hennebold JD, Grafe M, Colgin L, Lewis A, Ducore R, Swanson T, Legasse AW, Axthelm MK, MacAllister R, Moses AV, Morgan TK, Frias AE, Streblow DN. 2018. Zika virus infection in pregnant rhesus macaques causes placental dysfunction and immunopathology. Nat Commun 9:263.

3. Platt DJ, Smith AM, Arora N, Diamond MS, Coyne CB, Miner JJ. 2018. Zika virus-related neurotropic flaviviruses infect human placental explants and cause fetal demise in mice. Sci Transl Med 10.

4. Yockey LJ, Jurado KA, Arora N, Millet A, Rakib T, Milano KM, Hastings AK, Fikrig E, Kong Y, Horvath TL, Weatherbee S, Kliman HJ, Coyne CB, Iwasaki A. 2018. Type I interferons instigate fetal demise after Zika virus infection. Sci Immunol 3.

5. Casazza RL, Lazear HM. 2018. Antiviral immunity backfires: Pathogenic effects of type I interferon signaling in fetal development. Sci Immunol 3.

6. Gresser I, Morel-Maroger L, Riviere Y, Guillon JC, Tovey MG, Woodrow D, Sloper JC, Moss J. 1980. Interferon-induced disease in mice and rats. Ann N Y Acad Sci 350:12-20.

7. Cameo M, Fontana V, Cameo P, Vauthay LG, Kaplan J, Tesone M. 1999. Similar embryotoxic effects of sera from infertile patients and exogenous interferon-gamma on long-term in-vitro development of mouse embryos. Hum Reprod 14:959-63.

8. Ihara T, Oneda S, Yamamoto T, Boudrel L, Lau D, Miller D, Nagata R. 1999. An Embryotoxic/Teratogenic Potential and Abortifacient Effect Study of Interferon alfacon-1 (Infergen $\circledast$ ) via Subcutaneous Administration to Rhesus Monkeys. Congenital Anomalies 39:223-242.

9. Zusman I, Engelhard D, Yaffe P, Ron A, Panet A, Ornoy A. 1984. Effects of interferon and encephalomyocarditis virus on in vitro development of preimplantation mouse embryos with and without the zona pellucida. Teratology 29:405-9.

10. Desai TM, Marin M, Chin CR, Savidis G, Brass AL, Melikyan GB. 2014. IFITM3 restricts influenza A virus entry by blocking the formation of fusion pores following virusendosome hemifusion. PLoS Pathog 10:e1004048.

11. Li K, Markosyan RM, Zheng YM, Golfetto O, Bungart B, Li M, Ding S, He Y, Liang C, Lee JC, Gratton E, Cohen FS, Liu SL. 2013. IFITM proteins restrict viral membrane hemifusion. PLoS Pathog 9:e1003124.

12. Chesarino NM, Compton AA, McMichael TM, Kenney AD, Zhang L, Soewarna V, Davis M, Schwartz O, Yount JS. 2017. IFITM3 requires an amphipathic helix for antiviral activity. EMBO Rep 18:1740-1751. 
13. Yount JS, Moltedo B, Yang YY, Charron G, Moran TM, Lopez CB, Hang HC. 2010. Palmitoylome profiling reveals S-palmitoylation-dependent antiviral activity of IFITM3. Nat Chem Biol 6:610-4.

14. Yount JS, Karssemeijer RA, Hang HC. 2012. S-palmitoylation and ubiquitination differentially regulate interferon-induced transmembrane protein 3 (IFITM3)-mediated resistance to influenza virus. J Biol Chem 287:19631-41.

15. Percher A, Ramakrishnan S, Thinon E, Yuan X, Yount JS, Hang HC. 2016. Mass-tag labeling reveals site-specific and endogenous levels of protein S-fatty acylation. Proc Natl Acad Sci U S A 113:4302-7.

16. McMichael TM, Zhang L, Chemudupati M, Hach JC, Kenney AD, Hang HC, Yount JS. 2017. The palmitoyltransferase ZDHHC20 enhances interferon-induced transmembrane protein 3 (IFITM3) palmitoylation and antiviral activity. J Biol Chem 292:21517-21526.

17. McMichael TM, Zhang Y, Kenney AD, Zhang L, Zani A, Lu M, Chemudupati M, Li J, Yount JS. 2018. IFITM3 Restricts Human Metapneumovirus Infection. J Infect Dis doi:10.1093/infdis/jiy361.

18. Blaise S, de Parseval N, Benit L, Heidmann T. 2003. Genomewide screening for fusogenic human endogenous retrovirus envelopes identifies syncytin 2, a gene conserved on primate evolution. Proc Natl Acad Sci U S A 100:13013-8.

19. Blond JL, Lavillette D, Cheynet V, Bouton O, Oriol G, Chapel-Fernandes S, Mandrand B, Mallet F, Cosset FL. 2000. An envelope glycoprotein of the human endogenous retrovirus HERV-W is expressed in the human placenta and fuses cells expressing the type D mammalian retrovirus receptor. J Virol 74:3321-9.

20. Frendo JL, Olivier D, Cheynet V, Blond JL, Bouton O, Vidaud M, Rabreau M, EvainBrion D, Mallet F. 2003. Direct involvement of HERV-W Env glycoprotein in human trophoblast cell fusion and differentiation. Mol Cell Biol 23:3566-74.

21. Mi S, Lee X, Li X, Veldman GM, Finnerty H, Racie L, LaVallie E, Tang XY, Edouard P, Howes S, Keith JC, Jr., McCoy JM. 2000. Syncytin is a captive retroviral envelope protein involved in human placental morphogenesis. Nature 403:785-9.

22. Vargas A, Moreau J, Landry S, LeBellego F, Toufaily C, Rassart E, Lafond J, Barbeau B. 2009. Syncytin-2 plays an important role in the fusion of human trophoblast cells. J Mol Biol 392:301-18.

23. Chen CP, Chen LF, Yang SR, Chen CY, Ko CC, Chang GD, Chen H. 2008. Functional characterization of the human placental fusogenic membrane protein syncytin 2. Biol Reprod 79:815-23.

24. Kudo Y, Boyd CA. 2002. Changes in expression and function of syncytin and its receptor, amino acid transport system $\mathrm{B}(0)$ (ASCT2), in human placental choriocarcinoma BeWo cells during syncytialization. Placenta 23:536-41.

25. Borges M, Bose P, Frank HG, Kaufmann P, Potgens AJ. 2003. A two-colour fluorescence assay for the measurement of syncytial fusion between trophoblast-derived cell lines. Placenta 24:959-64.

26. Knerr I, Schubert SW, Wich C, Amann K, Aigner T, Vogler T, Jung R, Dotsch J, Rascher W, Hashemolhosseini S. 2005. Stimulation of GCMa and syncytin via cAMP mediated PKA signaling in human trophoblastic cells under normoxic and hypoxic conditions. FEBS Lett 579:3991-8.

27. Buchrieser J, Degrelle SA, Couderc T, Nevers Q, Disson O, Manet C, Donahue DA, Porrot F, Hillion KH, Perthame E, Arroyo MV, Souquere S, Ruigrok K, Dupressoir A, Heidmann T, Montagutelli X, Fournier T, Lecuit M, Schwartz O. 2019. IFITM proteins inhibit placental syncytiotrophoblast formation and promote fetal demise. Science 365:176-180.

28. Bayer A, Lennemann NJ, Ouyang Y, Bramley JC, Morosky S, Marques ET, Jr., Cherry S, Sadovsky Y, Coyne CB. 2016. Type III Interferons Produced by Human Placental 
Trophoblasts Confer Protection against Zika Virus Infection. Cell Host Microbe 19:70512.

29. Corry J, Arora N, Good CA, Sadovsky Y, Coyne CB. 2017. Organotypic models of type III interferon-mediated protection from Zika virus infections at the maternal-fetal interface. Proceedings of the National Academy of Sciences of the United States of America 114:9433-9438.

30. Thul PJ, Akesson L, Wiking M, Mahdessian D, Geladaki A, Blal HA, Alm T, Asplund A, Bjork L, Breckels LM, Backstrom A, Danielsson F, Fagerberg L, Fall J, Gatto L, Gnann C, Hober S, Hjelmare M, Johansson F, Lee S, Lindskog C, Mulder J, Mulvey CM, Nilsson P, Oksvold P, Rockberg J, Schutten R, Schwenk JM, Sivertsson A, Sjostedt E, Skogs M, Stadler C, Sullivan DP, Tegel H, Winsnes C, Zhang C, Zwahlen M, Mardinoglu A, Ponten F, von Feilitzen K, Lilley KS, Uhlen M, Lundberg E. 2017. A subcellular map of the human proteome. Science 356:820-+.

31. Chesarino NM, McMichael TM, Hach JC, Yount JS. 2014. Phosphorylation of the antiviral protein interferon-inducible transmembrane protein 3 (IFITM3) dually regulates its endocytosis and ubiquitination. J Biol Chem 289:11986-92.

32. Jia R, Pan Q, Ding S, Rong L, Liu SL, Geng Y, Qiao W, Liang C. 2012. The N-terminal region of IFITM3 modulates its antiviral activity by regulating IFITM3 cellular localization. J Virol 86:13697-707.

33. Melvin WJ, McMichael TM, Chesarino NM, Hach JC, Yount JS. 2015. IFITMs from Mycobacteria Confer Resistance to Influenza Virus When Expressed in Human Cells. Viruses 7:3035-52.

34. Battles MB, Langedijk JP, Furmanova-Hollenstein P, Chaiwatpongsakorn S, Costello HM, Kwanten L, Vranckx L, Vink P, Jaensch S, Jonckers TH, Koul A, Arnoult E, Peeples ME, Roymans D, McLellan JS. 2016. Molecular mechanism of respiratory syncytial virus fusion inhibitors. Nat Chem Biol 12:87-93.

35. Chesarino NM, McMichael TM, Yount JS. 2015. E3 Ubiquitin Ligase NEDD4 Promotes Influenza Virus Infection by Decreasing Levels of the Antiviral Protein IFITM3. PLoS Pathog 11:e1005095.

36. Lin TY, Chin CR, Everitt AR, Clare S, Perreira JM, Savidis G, Aker AM, John SP, Sarlah D, Carreira EM, Elledge SJ, Kellam P, Brass AL. 2013. Amphotericin B increases influenza A virus infection by preventing IFITM3-mediated restriction. Cell Rep 5:895908.

37. Moltedo B, Li W, Yount JS, Moran TM. 2011. Unique type I interferon responses determine the functional fate of migratory lung dendritic cells during influenza virus infection. PLoS Pathog 7:e1002345.

38. Yu J, Li M, Wilkins J, Ding S, Swartz TH, Esposito AM, Zheng YM, Freed EO, Liang C, Chen BK, Liu SL. 2015. IFITM Proteins Restrict HIV-1 Infection by Antagonizing the Envelope Glycoprotein. Cell Rep 13:145-156. 